\title{
Le Giornate Mondiali della Gioventů - i giovani e il beato Giovanni Paolo II
}

\section{The World Youth Day - the Young and the Blessed John Paul II}

\begin{abstract}
Many people say that World Youth Day is Pope John Paul II's best invention. However, he used to say that it was the young people themselves who invented it. The Pope felt that the Church's concern for the younger generations should be made known. He issued invitation to young people to come to Rome for Palm Sunday. After two gatherings, at the end of 1985 he announced the institution of the World Youth Day that was to be celebrated every year in the dioceses. The diocesan celebrations were soon joined by international gatherings. The author of this article presents World Youth Day as the Church's Day for youth and with youth. This idea is not an alternative to ordinary youth ministry, often carried out with great sacrifice and self-denial. Indeed it intends actually to consolidate this work by offering new encouragement for commitment, objectives which foster ever greater involvement and participation. By aiming to foster greater fervour in apostolate among young people, the Church desires to make them the protagonists of an apostolate which will spread to the other ages and situations of life in the ambit of new evangelization.
\end{abstract}

\section{Keywords}

The World Youth Day, John Paul II, the youth.

Per anni la stampa ostile alle iniziative di Giovanni Paolo II parlava delle Giornate Mondiali della Gioventù come di Woodstock cattolica, oppure di dimostrazione di forza da parte di un Papa conservatore in grado di mobilitare 
le sue falangi di giovani crociati cattolici. Anche all'interno della Chiesa alcuni parlavano di un evento che non passerà alla storia, perché destinato a rimanere alla superficie delle cose, perciò effimero.

Beato Giovanni Paolo II però aveva un programma. È partito con la precisa finalità principale delle Giornate Mondiali della Gioventù. Per Lui e per i suoi collaboratori le Giornate dovevano riportare al centro della fede e della vita di ogni giovane la persona di Gesù, perché ne diventi costante punto di riferimento e perché sia anche la vera luce di ogni iniziativa e di ogni impegno educativo verso le nuove generazioni. È il "ritornello" di ogni Giornata Mondiale. E tutte insieme, nell'arco di quasi trentanni, appaiono come un continuo e pressante invito a fondare la vita e la fede sulla roccia che è Cristo. Col passare degli anni, le Giornate Mondiali della Gioventù hanno confermato di non essere riti convenzionali, ma eventi provvidenziali, occasioni per i giovani di professare e proclamare con crescente gioia la fede in Cristo. Ritrovandosi, essi possono interrogarsi insieme sulle aspirazioni più intime, sperimentare la comunione con la Chiesa, impegnarsi nell'urgente compito della nuova evangelizzazione. In tal modo si danno la mano, formando un immenso cerchio di amicizia, congiungendo i colori della pelle e delle bandiere nazionali, la varietà delle culture e delle esperienze, nell'adesione di fede al Signore Risorto'

Senza dubbio le Giornate Mondiali della Gioventù sono un segno, un luogo e un grande passo avanti nella nuova evangelizzazione tra i giovani. Ma per capirle meglio dobbiamo tornare all'inizio. Tra gli eventi che hanno preparato i terreno per questa risoluzione bisogna ricordare il Giubileo dei Giovani del 1984, incentrato sul tema: Aprite le porte al Redentore. Sul invito del Papa, arrivarono a Roma da tutto il mondo migliaia e migliaia di giovani. Cardinale Stanisław Ryłko così ricorda questi eventi: "Chi era rimasto ai tempi della grande contestazione degli anni Sessanta e Settanta restò sbalordito dinanzi a quel segno: nel mondo dei giovani stava verificandosi qualcosa di nuovo, cioè un riavvicinamento alla fede e alla Chiesa"2. L'anno seguente, il 1985, fu poi proclamato dalle Nazioni Unite Anno internazionale della gioventù. A Roma ha luogo un altro grande incontro del Papa con giovani di tutto il mondo. Ma soprattuto nello stesso anno Giovanni

${ }^{1}$ Cf. Jan Paweł II, Znaczenie Światowych Dni Młodzieży, List Ojca Świętego do kard. Eduardo Francisco Pironio z okazji seminarium zorganizowanego w dniach 13-16 maja 1996 r. w Częstochowie (Lettera di Giovanni Paolo II al Cardinale Eduardo Francisco Pironio in occasione del Seminario di studio sulle Giornate Mondiali della Gioventù promosso a Częstochowa), „L'Osservatore Romano” (ed. pol.), 7-8 (1996), p. 4-5.

${ }^{2}$ S. Ryłko, I giovani e il Papa. Insieme, in: Pontificium Consilium pro Laicis, Insiemie sulle strade dell'Europa, III Convegno Europeo di Pastorale Giovanile, Paderborn, 21-24 settmbre 1998. Città del Vaticano 1999, p. 176. 
Paolo II pubblica la Lettera ai giovani e alle giovani del mondo, chiamata fino d'oggi da molti responsabili della pastorale giovanili "enciclica dei giovani"’3.

Dalla prima Giornata Mondiale della Gioventù, essa costituisce la giornata della Chiesa per i giovani e con i giovani. Come ha sottolineato beato Giovanni Paolo II la proposta delle GMG non si pone in alternativa della pastorale giovanile svolta ordinariamente, spesso con grande sacrificio e abnegazione. Essa vuole piuttosto rinsaldarla offrendole nuovi stimoli d'impegno, mete sempre più coinvolgenti e partecipate. Puntando a suscitare crescente fervore nell'azione apostolica tra i giovani, non vuole certo isolarli dal resto della comunità, bensì renderli protagonisti di un apostolato che contagi le altre età e situazioni di vita nell'ambito della nuova evangelizzazione. I vari momenti in cui si articola una Giornata Mondiale costituiscono nel loro insieme una sorta di vasta catechesi, un annuncio del cammino di conversione a Cristo, a partire dalle esperienze e dagli interrogativi profondi della vita quotidiana dei destinatari. La Parola di Dio ne è il centro, la riflessione catechistica lo strumento, la preghiera l'alimento, la comunicazione e il dialogo lo stile 4 .

\section{GMG è forma necessaria e aggiornata di evangelizzazione}

Per Giovanni Paolo II l'uomo - in questo caso il giovane - era la fondamentale ed insieme quotidiana via della Chiesa (Redemptor Hominis, 14), allora si comprende bene perché lui attribuiva una speciale importanza al periodo della giovinezza come ad una tappa-chiave della vita di ogni uomo. Per lui giovani erano la giovinezza delle nazioni e delle società, la giovinezza di ogni famiglia e dell'intera umanità; anche la giovinezza della Chiesa.

"In voi c'è la speranza, perché voi appartenete al futuro, come il futuro appartiene a voi. La speranza, infatti, è sempre legata al futuro, è l'attesa dei «beni futuri». Come virtù cristiana, essa è unita all'attesa di quei beni eterni, che Dio ha promesso all'uomo in Gesù Cristo 5 . E contemporaneamente questa speranza, come virtù insieme cristiana e umana, è l'attesa dei beni che l'uomo si costruirà utilizzando i talenti a lui dati dalla Provvidenza. In questo senso a voi, giovani, appartiene il futuro, così come un tempo esso appartenne alla generazione degli

3 A. Kiciński, Historia Papieskiej Szkoty Modlitwy (La storia della pontificia scuola di preghiera), in: S. Kulpaczyński (ed.), Modlitwa w katechezie (Pregiera nella catechesi), Lublin 2002, p. 331-381.

${ }^{4}$ Cf. Jan Paweł II, Znaczenie Światowych Dni Młodzieży, n. 3.

${ }^{5}$ Cfr. Rm 8,19.21; Ef 4,4; Fil 3,10s; Tm 3,7; Eb 7,19; 1 Pt 1,13. 


\section{The Person and the Challenges \\ 124

adulti e proprio insieme con essi è divenuto attualità. Di questa attualità, della sua molteplice forma e profilo sono responsabili prima di tutto gli adulti. A voi spetta la responsabilità di ciò che un giorno diventerà attualità insieme con voi, ed ora è ancora futuro. Quando diciamo che a voi appartiene il futuro, pensiamo in categorie di transitorietà umana, la quale è sempre un passaggio verso il futuro. Quando diciamo che da voi dipende il futuro, pensiamo in categorie etiche, secondo le esigenze della responsabilità morale, che ci ordina di attribuire all'uomo come persona - e alle comunità e società che sono composte da persone - il valore fondamentale degli atti, dei propositi, delle iniziative e delle intenzioni umane. Questa dimensione è anche la dimensione propria della speranza cristiana e umana" .

Giovanni Paolo II quasi sfidando i risultati delle ricerche sociologiche e dei sontaggi che dipingevano a fosche tinte la gioventù. Lui vedeva nei giovani un immenso potenziale di bene e di possibilità creative e aveva fiducia nei giovani, e i giovani lo sentivano. In essi vedeva una grande forza profetica. Non solo per il papa Wojtyła l'età giovanile è sempre connotata dall'anelito agli ideali, alla verità, alla bontà e alla bellezza, alla giustizia e alla solidarietà. È l'età che precede e prepara gli anni delle decisioni e delle scelte. Per lui era un segno che il mondo troppo spesso cede alla tentazione di sottovalutare le potenzialità di bene insite nei giovani.

Ma il Papa Giovanni Paolo II era sensibile ai segni dei tempi. Dopo due grandi incontri con i giovani, nel 1984 e 1985, egli istituisce nella Chiesa la Giornata Mondiale della Gioventù. La logica di ferro dall'inizio del Pontificato, che tutti i giovani devono sentirsi seguiti dalla Chiesa. Prima spiega la sua scelta ai responsabili della pastorale giovanile: "L'esperienza delle Giornate Mondiali invita tutti noi, Pastori ed operatori della pastorale, a riflettere costantemente sul nostro ministero in mezzo ai giovani e sulla responsabilità che abbiamo di presentare loro la verità piena su Cristo e sulla sua Chiesa. Come non leggere nella loro partecipazione massiccia, disponibile ed entusiasta la costante richiesta di essere accompagnati nel pellegrinaggio di fede, nel viaggio che compiono in risposta alla grazia di Dio operante nei loro cuori? Essi si rivolgono a noi perché li conduciamo a Cristo che, solo, ha parole di vita eterna ${ }^{7}$. Ascoltare i giovani e insegnare loro richiede attenzione, tempo e sapienza. La pastorale giovanile costituisce una delle priorità della Chiesa alle soglie del terzo millennio. Con il loro entusiasmo e la loro esuberante energia, i giovani chiedono di essere incoraggiati a diventare «protagonisti dell'evangelizzazione e artefici del

\footnotetext{
${ }^{6}$ Lettera ai giovani, 1985, n. 1.

${ }^{7}$ Cf. Gv 6, 68.
} 
rinnovamento sociale $»^{8}$. In tal modo i giovani, nei quali la Chiesa riconosce la sua stessa giovinezza di Sposa di $\mathrm{Cristo}^{9}$, non solo vengono evangelizzati, ma diventano essi stessi evangelizzatori che portano il Vangelo ai loro coetanei, compresi quanti sono estranei alla Chiesa e non hanno ancora udito la Buona Novella" ${ }^{10}$. Egli stesso afferma questa scelta più tardi nel libro Varcare la soglia della speranza: "Nessuno ha inventato le Giornate Mondiali dei Giovani. Furono proprio loro a crearle. Quelle Giornate, quegli incontri, divennero da allora bisogno dei giovani di tutti i luoghi del mondo. Il più delle volte sono state una grande sorpresa per i pastori, e persino per i vescovi. Hanno superato quanto anch'essi si aspettavano"11.

La Giornata Mondiale della Gioventù si è evoluta come una forte e coinvolgente esperienza di fede che vede, fino d'oggi, protagonisti i giovani con tutta la carica di novità e di futuro che essi esprimono e testimoniano nella Chiesa e nel mondo intero. I partecipanti hanno obiettivi precisi: incontrarsi, crescere insieme attraverso scambi di esperienze, pregare con il Papa. E fare di quei giorni una grande festa che rappresenti l'immagine di credere insieme. Ma non solo. Si tratta infatti di un vero e proprio pellegrinaggio ${ }^{12}$ e non certo di una semplice kermesse di giovani cattolici, né tantomeno una sorta di avvenimento turisticoreligioso, sebbene non manchino spazi dedicati alla cultura e allo svago.

I giovani che accorrevano a questi importanti appuntamenti con Giovanni Paolo II sono stati sempre più numerosi: dopo Buenos Aires, si sono rivisti a Santiago de Compostela, in Spagna, nel 1989; poi a Częstochowa, in Polonia nel 1991. Due anni dopo sono arrivati tra le montagne rocciose di Denver, negli Stati Uniti; si sono spinti fin nel cuore dell'Asia, a Manila, nel 1995; sono accorsi numerosi sotto la Torre Eiffeil, a Parigi nel 1997. A Roma 2000 le Giornate Mondiali hanno raggiunto il culmine di fede e di speranza con più di 2 milioni di giovani che credendo in Gesù Cristo hanno cantato tutti insieme "sotto la stessa luce sotto la sua Croce". Sulle orme di Roma Giovanni Paolo II ci ha dato appuntamento in Canada nel 2002 e Toronto per la XVII Giornata Mondiale della Gioventù, che è stata la sua ultima Giornata terrena. Ogni GMG con Giovanni Paolo II ha avuto

\footnotetext{
${ }^{8}$ Christifideles laici, 46.

${ }^{9}$ Cf. Ef 5, 22-33.

${ }^{10}$ Cf. Jan Paweł II, Znaczenie Światowych Dni Młodzieży, n. 4.

${ }^{11}$ Jan Paweł II, Przekroczyć próg nadziei (Varcare la soglia della speranza), Lublin 1994, p. 103-104.

${ }^{12}$ Cf. Servizio Nationale per la Pastorale Giovanile della Conferenza Episcopale Italiana, Giovani e pellegrini, Roma 1995.
} 
la sua storia irrepetibile, ed è stata una pietra miliare della storia spirituale della gioventù del nostro tempo.

\section{GMG con la gioventủ reale in una continua trasformazione}

Beato Giovanni Paolo II vedeva i giovani che sono desiderosi di affidarsi a qualcuno, ma non sanno a chi raccontare se stessi. Egli si rendeva conto che poche sono le esperienze di amore incondizionato che giovani incontrano sul loro cammino. Per questo scriveva a loro: "Il Papa pensa a voi e vi vuole bene, vi raggiunge quotidianamente con un pensiero carico d'afetto e vi accompagna con la preghiera, si fida e conta su di voi, sul vostro impegno cristiano e sulla vostra collaborazione alla causa del bene"13. Il Papa sapeva che durante il suo pontificato nei Paesi dell'Est e del Sud del mondo la domanda religiosa dei giovani è sempre stata una costante, dove era naturale pensare a una fede, naturale rifarsi a Dio e orientale la propria vita ai valori religiosi. Ma egli già vedeva che contrariamente a qualche decennio prima, anche nei Paesi occidentali, i giovani tornano a porsi domande religiose. Che sono sensibili a esperienze forti, eccezionali, capaci di far superare atteggiamenti di indifferenza che sembravano persistere in certi ambienti secolarizzati.

La diagnosi che Giovanni Paolo II faceva della gioventù era reale. Egli avvertiva forte quando giovani nella ricerca religosa e spirituale rischiavano di perdersi in un nuovo paganesimo, in movimenti confusi e deludenti come la New Age o esperienze magiche e settarie. Dalle sue parole si sentiva che i giovani sperimentano spesso la sua fragilità e solitudine di fronte allo sviluppo e al consumo. Lui sottolineva che per i paesi industrilizzati l'esorbiente numero di occassioni, di proposte, di iniziative, di offerte di beni materiali minacciava gravemente la capacità di scegliere, di decidere, di orientarsi. Con preoccupazione osservava che per loro è difficile trovare riferimenti morali soprattutto riguardo alla vita affettiva e sessuale. Nello stesso tempo il Papa vedeva che negli altri Paesi la fragilità dei giovani è accentuata dalla mancanza di beni indispensabili per una vita dignitosa mentre il consumismo dell'Occidente, offesa a tanta povertà, propone modelli di vita che spesso distolgono le migliori energie da obiettivi di crescita umana, culturale e sociale.

Il Papa coglieva una forte esigenza di radicalità dei giovani. Notava il clima di libertà che caratterizzava le nuove generazioni nei confronti degli adulti e delle ideologie e che portava i giovani a porsi domande religiose, a compiere scelte più

\footnotetext{
${ }^{13}$ Messaggio ai giovani, 1998 n. 1.
} 
consapevoli e quidi a volere il massimo dell'esperienza religosa. Cammino delle GMG permette a notare che il Papa sapeva che i giovani non si accontentano di appartenere sociologicamente a un mondo di pensare, a dei riti collettivi, a delle abitudini e tradizioni, ma vogliono cogliere il centro della esperienza religiosa. Giovanni Paolo II continuando il dialogo con i giovani leggeva che per loro la figura di Gesù Cristo esercitava ancora un fascino particolare per coloro che vivevano negli ambienti legati alla comunità cristiana o che ritornavano alla fede dopo averla abbandonata nella prima adolescenza. Nella continua trasformazione del mondo giovanile gli aiutava nella ricerca di un Padre che faccia breccia nei cuori e vinca l'insicurezza e la solitudine ${ }^{14}$.

\section{GMG per la crescita umana e cristiana}

Le Gioranate Mondiali hanno dimonstrato un raduno centinaia di migliaia e perfino milioni di partecipanti, di persone non «masse». Cosa ha fatto beato Giovanni Paolo II che era così? Si può rispondere a questa domanda con la Lettera Apostolica ai giovani e alle giovani del mondo del 1985 e con centinaia altri discorsi. Il Papa ha impostato tutti gli incotri con i giovani nel ottica del dialogo tra ogni giovane con Gesù Cristo. "Avvertiremo questo rapporto in modo forse più distinto, quando mediteremo il colloquio di Cristo col giovane, riferito dagli evangelisti ${ }^{15}$. Tra i molti testi biblici è questo prima di tutto che merita di essere qui ricordato"16. Questa osservazione viene conferemata dal forte accento esperienziale di questo dialogo prezente nelle Giornate Mondiali: "Egli chiama ciasuno di noi (...). Fin dall'eternità Dio ha pensato a noi e ci ha amati come persone uniche e irripetibili"' ${ }^{17}$. Come afferma Guzmán Carriquiry Lecour, SottoSecretario dal 1991 al 2011 del Pontificio Consiglio per i Laici, così il rapporto educativo procede da una esperienza vissuta insieme ma che non rimane in una logica di gruppo. Si rivolge ad ogni persona, alla sua libertà. Perché al Papa Wojtyła interessava solo il valore della persona, l'adesione della sua libertà, 1"'eccomi" di fronte alla chiamata per nome, il fatto di venir comunicato da

${ }^{14}$ Cf. A. Kiciński, Tożsamość ludzi młodych w nauczaniu Jana Pawła II podczas pielgrzymek do Polski (Identità dei giovani nell'insegnamento di Giovanni Paolo II), in: S. Kulpaczyński (ed.), Katecheza młodych (Catechesi dei giovani), Lublin 2003, p. 271-292.

${ }^{15}$ Cfr. Mc 10,17-22; Mt 19,16-22; Lc 18,18-23.

${ }^{16}$ Giovanni Paolo II, Lettera Apostolica ai giovani e alle giovani del mondo, n. 2.

${ }^{17}$ Giovanni Paolo II, Veglia, Santiago de Compostela, 19.08.1989. 
persona a persona, di esperienza in esperienza. La personalizzazione della fede nell'ambito di un mistero di comunione ${ }^{18}$.

Rimane la traccia di riflessione fondamentale circa la formazione cristiana proposta da Giovanni Paolo II ai giovani durante le Giornate Mondiali circa il bisogno essenziale che loro avevano di forti testimonianze per la crescita umana e cristiana. Nella condizione giovanile sono fondamentali le testimonianze di altri giovani, che dimonstrino con la loro vita che Gesù Cristo è la chiave di risposta reale ai bisogni e ai desideri che si presentano sempre nell'esistenza giovanile. Ma non si cresce solo con l'emulazione fraterna ma nel riferimento alle figure paterna e materna. Giovanni Paolo II sapeva che i giovani rifiutano ogni "paternalismo", che non fosse una autentica paternità, cioè un'autorità nel senso etimologico e più vero del termine: ciò che coltiva e fa crescere l'umanità dell'uomo. Il Papa è stato percepito dai giovani come testimone per eccelenza, e periò riconosciuto come maestro ${ }^{19}$.

\section{GMG nella valutazione pastorale di Benedetto XVI}

Ma come viene valutata dal punto di vista pastorale odierna l'intuizione che portò Papa Wojtyła a promuovere i grandi raduni giovanili che si sono susseguiti nel Pontificato di Ratzinger?

Uno studio di Lucio Meglio ${ }^{20}$ mette in rilievo il fatto che, con la morte di Giovanni Paolo II, egli ha lasciato una pesante eredita al suo successore: il cardinale tedesco Joseph Ratzinger. Di carattere diametralmente opposto al suo predecessore, il nuovo pontefice si è trovato a dover obbligatoriamente percorrere i passi già tracciati nel rapporto con le nuove generazioni da papa Wojtyła; indietro non si poteva tornare, e la Giornata mondiale della gioventu di Colonia ne è stata il banco di prova. Autore mette in chiaro le differenze tra $\mathrm{i}$ due pontefici. La sua ricerca dimostra che nel primo anno di pontificato Giovanni Paolo II dedicò diciotto discorsi ai più giovani, Benedetto XVI otto, meno della metà. Il suo lavoro basato interamente sullo studio esplorativo e descrittivo delle parole o unità d'analisi elementari, intese come eventi di "microstoria" capaci di

${ }^{18}$ Cf. G. Carriquiry Lecour, Le Giornate Mondiali della Gioventù, in: Pontificio Consiglio per i Laici, Insieme sulle strade dell'Europa, Atti del II Convegno Europeo di Pastorale Giovanile, Loreto, 12-16 settembre 1995, Città del Vaticano 1996, p. 65.

${ }^{19}$ Idem.

${ }^{20}$ L. Meglio, L'attegiamento nei confronti dei giovani di Giovanni Paolo II e Benedetto XVI. Analisi del contenuto dei discorsi dei due pontefici, http://www.religioniesocieta.it/upload/dl/ ASFeR/lmeglio_2papi.pdf (15.04.2012). 
illustrare la "macrostoria" da cui derivano. Questo che ci sembra importante è confrontare la guaduatoria delle parole più ricorrenti nei due discorsi.

Tab. Prime 10 parole con relative frequenze

\begin{tabular}{|c|c|c|c|}
\hline \multicolumn{2}{|c|}{ Giovanni Paolo II } & \multicolumn{2}{c|}{ Benedetto XVI } \\
\hline Parole & Frequenze & Parole & Frequenze \\
\hline Giovani & 413 & Dio & 178 \\
\hline Cristo & 339 & Vita & 76 \\
\hline Dio & 264 & Cristo & 69 \\
\hline Gesù & 191 & Gesù & 69 \\
\hline Uomo & 172 & Mondo & 55 \\
\hline Mondo & 169 & Amore & 55 \\
\hline Amore & 151 & Cuore & 50 \\
\hline Chiesa & 142 & Uomo & 54 \\
\hline Verità & 121 & Giovani & 46 \\
\hline Fede & 94 & Amici & 46 \\
\hline
\end{tabular}

Dallo studio di L. Melgio appare evidente che analizzando solo le prime due parole compare già un indice abbastanza indicativo del rapporto tra i due pontefici e l'universo giovanile. La parola più ricorrente nei discorsi di papa Wojtyła è giovani, in Ratzinger è Dio.

Conoscendo il cardinale Ratzinger si poteva aspettare qualche ridimensionamento del ruolo delle GMG nella storia recente della Chiesa. Al sorpresa nel discorso tenuto al tradizionale incontro degli auguri natalizi alla Curia romana nella Sala Clementina, Papa Benedetto XVI ha parlato ampiamente della "magnifica esperienza della Giornata Mondiale della Gioventù" come di una "medicina contro la stanchezza del credere", una "nuova evangelizzazione vissuta". Il Papa Ratzinger, dopo l'esperienza della GMG di Madrid, delinea nelle Giornate Mondiali della Gioventù un modo nuovo, ringiovanito, dell'essere cristiani che tenta di caratterizzare in cinque punti.

Nel primo posto, mette Benedetto XVI, una nuova esperienza della cattolicità, dell'universalità della Chiesa. È questo che lo colpisce in modo immediato. Partecipanti della GMG provenienti da tutti i continenti, e, pur non essendoci mai visti prima, si riconoscono cristiani. Pur parlando lingue diverse e avendo differenti abitudini di vita, differenti forme culturali, e tuttavia ci si trovano subito uniti insieme come una grande famiglia. Separazione e diversità esteriori sono relativizzate. Mette in luce l'esperienza della GMG dove tutti comprendono anche in modo molto concreto che, nonostante tutte le fatiche e le oscurità, è 


\section{The Person and the Challenges \\ 130

bello appartenere alla Chiesa universale, alla Chiesa cattolica, che il Signore ci ha donato.

Nel secondo posto vede il servizio dei volontari, che mettono a disposizione settimane o mesi della loro vita per collaborare alle preparazioni tecniche, organizzative e contenutistiche della Giornata Mondiale della Gioventù e proprio così rendono possibile lo svolgimento ordinato del tutto. Con il proprio tempo l'uomo dona sempre una parte della propria vita. Per Ratzinger, questi giovani sono visibilmente e "tangibilmente" colmi di una grande sensazione di felicità: il loro tempo donato aveva un senso; proprio nel donare il loro tempo e la loro forza lavorativa avevano trovato il tempo, la vita. E allora per Lui è diventata evidente una cosa fondamentale: questi giovani avevano offerto nella fede un pezzo di vita, non perché questo era stato comandato e non perché con questo ci si guadagna il cielo; neppure perché così si sfugge al pericolo dell'inferno. Non l'avevano fatto perché volevano essere perfetti. Questi giovani hanno fatto del bene - anche se quel fare è stato pesante, anche se ha richiesto sacrifici - semplicemente perché fare il bene è bello, esserci per gli altri è bello.

Gli altri elementi per il Papa Ratzinger, che fano parte delle Giornate Mondiali della Gioventù e della spiritualità da esse proveniente, sono: l'adorazione (la novità di Ratzinger), la presenza del Sacramento della Penitenza e infine, come ultima caratteristica da non trascurare nella spiritualità delle Giornate Mondiali della Gioventù la gioia. La fede rende lieti è questa una delle esperienze meravigliose delle Giornate Mondiali della Gioventù ${ }^{21}$. Dopo la morte del Papa delle GMG il nuovo Pontefice Joseph Ratzinger non solo ha accolto i giovani nella sua terra di origine, in Germania e precisamente a Colonia, nell'agosto del 2005 ma in linea principale con il suo predecessore, Benedetto XVI vede nelle GMG un incontro con Cristo.

Per tanti giovani la GMG con Giovanni Paolo II è diventata una specie di «laboratorio della fede», il luogo della riscoperta di una fede e di una religiosità che non sono in contrasto con l'essere giovani. Durante questi incontri sono nate tante vocazioni al sacerdozio e alla vita consacrata. C'è chi afferma che nel mondo

${ }^{21}$ Benedetto XVI, Discorso del Santo Padre agli Em.mi Signori Cardinali, alla Curia Romana e alla Famiglia Pontificia, per la presentazione degli Auguri Natalizi, http://www.vatican.va/ holy_father/benedict_xvi/speeches/2011/december/documents/hf_ben-xvi_spe_20111222_auguricuria_it.html (15.04.2012). 
dei giovani è in atto una "rivoluzione silenziosa", il cui potente motore propulsore sono proprio le GMG. È grazie alle Giornate Mondiali della Gioventù che la Chiesa, alle soglie del terzo millennio con un vecchio Papa Wojtyła, ha ritrovato il suo volto giovane, il volto dell'entusiasmo e di un coraggio rinnovato. La storia delle GMG è la storia affascinante della nascita di una nuova generazione di giovani: giovani del «sì» a Cristo, dell'adesione convinta alla Chiesa e al Papa. Giovanni Paolo II li chiamava "sentinelle del mattino" (Roma, 2000), "popolo delle beatitudini" (Toronto, 2002) ${ }^{22}$.

Ci sono stati e ci sono le voci di critica delle Giornate Mondiali della Gioventù. Il problema "the day after" inutile nasconderlo: un fantasma alleggia sulle Giornate Mondiali, come su tutte le manifestazioni religiose di massa. Nonostante il successo quantitativo e qualitativo che questo tipo di esperienze può avere, resta sempre negli osservatori il sospetto che si tratti di momenti fuggevoli. Ogni nuova edizione della GMG fa ritornare la domanda su quale sia il segreto di questo sorprendente fenomeno che rivela al mondo un volto del tutto inaspettato, non solo della Chiesa, ma degli stessi giovani d'oggi.

Senz dubbio intuizione del beato Giovanni Paolo II ha permesso di far incontrare milioni dei giovani con Cristo e con la sua Chiesa. Dal punto scientifico si può parlare di una teologia dei giovani nata durante questi incotri. Ma soprattutto dal punto di vista umano il carisma di Giovanni Paolo II di dialogare con i giovani ci ha colpito tutti. $\mathrm{Ci}$ ha fatto capire che si può amare i giovani e si può essere sensibile ai loro problemi. Si può osare a dire che le GMG sono un dono che continua a suscitare stupore all'interno della Chiesa e fuori di essa. E sono una fotografia di una gioventù molto diversa dal cliché diffuso dai media, ci mostrano una gioventù assetata di valori autentici e alla ricerca di un senso più profondo della vita.

\section{Il cammino delle Giornate Mondiali della Gioventù}

1984 Roma: Piazza S. Pietro, Domenica delle Palme (15 aprile 1984)

Grande raduno dei giovani in occasione dell'Anno Santo della Redenzione

Il Papa consegna la Croce ai giovani (22 aprile 1984)

${ }^{22} \mathrm{~S}$. Ryłko, Un fenomeno straordinario che dura da venticinque anni, http://www.laici.va/ content/dam/laici/documenti/giovani/articoli\%20d\%27interesse/italiano/intervento-del-cardrylko-sulla-gmg-2011.pdf (on-line 15.04.2012). 
1985 Roma: Piazza S. Pietro, Domenica delle Palme (31 marzo 1985)

Grande raduno dei giovani in occasione dell'Anno Internazionale della Gioventù Il Papa dedica una Lettera Apostolica ai giovani e alle giovani del mondo (31 marzo 1985) e poi annuncia l'istituzione della Giornata Mondiale della Gioventù (20 dicembre 1985)

\section{I Giornata Mondiale della Gioventù}

Tema: "Sempre pronti a rispondere a chiunque vi domandi ragione della speranza che è in voi" (1 Pt 3,15$)$

Celebrazione (diocesana): Domenica delle Palme (23 marzo 1986)

\section{II Giornata Mondiale della Gioventù}

Tema: "Noi abbiamo conosciuto e creduto all'amore che Dio ha per noi" $(1 \mathrm{Gv} 4,16)$

Celebrazione (internazionale): Buenos Aires, Argentina (11-12 aprile 1987)

\section{III Giornata Mondiale della Gioventù}

Tema: "Fate quello che Egli vi dirà" (Gv 2,5)

Celebrazione (diocesana): Domenica delle Palme (27 marzo 1988)

\section{IV Giornata Mondiale della Gioventù}

Tema: "Io sono la via, la verità e la vita" (Gv 14,6)

Celebrazione (internazionale): Santiago de Compostela, Spagna (15-20 agosto 1989)

\section{V Giornata Mondiale della Gioventù}

Tema: "Io sono la vite voi i tralci” (Gv 15,5)

Celebrazione (diocesana): Domenica delle Palme (8 aprile 1990)

\section{VI Giornata Mondiale della Gioventù}

Tema: "Avete ricevuto uno spirito da figli” $(\mathrm{Rm} 8,15)$

Celebrazione (internazionale): Czestochowa, Polonia (10-15 agosto 1991)

\section{VII Giornata Mondiale della Gioventù}

Tema: “Andate in tutto il mondo e predicate il vangelo" (Mc 16,15)

Celebrazione (diocesana): Domenica delle Palme (12 aprile 1992)

\section{VIII Giornata Mondiale della Gioventù}

Tema: "Io sono venuto perché abbiano la vita e l'abbiano in abbondanza"

(Gv 10,10)

Celebrazione (internazionale): Denver, USA (10-15 agosto 1993) 
1994-1995

Tema: "Come il Padre ha mandato me, anch'io mando voi" (Gv 20,21)

IX Giornata Mondiale della Gioventù

Celebrazione (diocesana): Domenica delle Palme (27 marzo 1994)

X Giornata Mondiale della Gioventù

Celebrazione (internazionale): Manila, Filippine (10-15 gennaio 1995)

\section{XI Giornata Mondiale della Gioventù}

Tema: "Signore, da chi andremo? Tu solo hai parole di vita eterna" (Gv 6,68)

Celebrazione (diocesana): Domenica delle Palme (31 marzo 1996)

\section{XII Giornata Mondiale della Gioventù}

Tema: "Maestro, dove abiti? Venite e vedrete" (Gv 1,38-39)

Celebrazione (internazionale): Parigi, Francia (19-24 agosto 1997)

\section{XIII Giornata Mondiale della Gioventù}

Tema: "Lo Spirito Santo vi insegnerà ogni cosa” (Gv 14,26)

Celebrazione (diocesana): Domenica delle Palme (5 aprile 1998)

\section{XIV Giornata Mondiale della Gioventù}

Tema: "Il Padre vi ama” (Gv 16,27)

Celebrazione (diocesana): Domenica delle Palme (28 marzo 1999)

\section{XV Giornata Mondiale della Gioventù}

Tema: "Il Verbo si fece carne e venne ad abitare in mezzo a noi" (Gv 1,14)

Celebrazione (internazionale): Roma (15-20 agosto 2000)

\section{XVI Giornata Mondiale della Gioventù}

Tema: "Se qualcuno vuol venire dietro a me, rinneghi se stesso, prenda la sua croce ogni giorno e mi segua” (Lc 9,23)

Celebrazione (diocesana): Domenica delle Palme (8 aprile 2001)

\section{XVII Giornata Mondiale della Gioventù}

Tema: "Voi siete il sale della terra... Voi siete la luce del mondo" (Mt 5, 13-14)

Celebrazione (internazionale): Toronto (23-28 luglio 2002)

\section{XVIII Giornata Mondiale della Gioventù}

Tema: “Ecco la tua Madre!” (Gv 19,27)

Celebrazione (diocesana): Domenica delle Palme (13 aprile 2003) 


\section{XIX Giornata Mondiale della Gioventù}

Tema: "Vogliamo vedere Gesù" (Gv 12,21)

Celebrazione (diocesana): Domenica delle Palme (4 aprile 2004)

\section{XX Giornata Mondiale della Gioventù (Benedetto XVI)}

Tema: "Siamo venuti per adorarlo" (Mt 2,2)

Celebrazione (internazionale): Colonia (16-21 agosto 2005)

\section{XXI Giornata Mondiale della Gioventù}

Tema: "Lampada per i miei passi è la tua parola, luce sul mio cammino"

(Sal 119, 105)

Celebrazione (diocesana): Domenica delle Palme (9 aprile 2006)

\section{XXII Giornata Mondiale della Gioventù}

Tema: "Come io vi ho amato, così amatevi anche voi gli uni gli altri" (Gv 13, 34)

Celebrazione (diocesana): Domenica delle Palme (8 aprile 2007)

\section{XXIII Giornata Mondiale della Gioventù}

Tema: "Avrete forza dallo Spirito Santo che scenderà su di voi e mi sarete testimoni” (At 1,8)

Celebrazione internazionale - Sydney, 15-20 luglio 2008

\section{XXIV Giornata Mondiale della Gioventù}

Tema: "Abbiamo posto la nostra speranza nel Dio vivente" $(1 \mathrm{Tm} 4,10)$

Celebrazione (diocesana): Domenica delle Palme (5 aprile 2009)

\section{XXV Giornata Mondiale della Gioventù}

Tema: "Maestro buono, che cosa devo fare per avere in eredità la vita eterna?"

(Mc 10,17)

Celebrazione (diocesana): Domenica delle Palme (28 marzo 2010)

\section{XXVI Giornata Mondiale della Gioventù}

Tema: "Radicati e fondati in Cristo, saldi nella fede" (cfr. Col 2,7)

Celebrazione internazionale - Madrid (Spagna), 16-21 agosto 2011

\section{XXVII Giornata Mondiale della Gioventù}

Tema: "Siate sempre lieti nel Signore!" (Fil 4,4)

Celebrazione (diocesana): Domenica delle Palme (1 aprile 2012) 